\title{
Distal Femoral Locking Compression Plate Fixation in Distal Femoral Fractures: Early Results
}

\author{
EJ Yeap, MS (Ortho)*, AS Deepak, MS (Ortho)** \\ *Department of Orthopaedic Surgery and Traumatology, Hospital Tuanku Fauziah, 01000 Kangar, Malaysia \\ **Department of Orthopaedic Surgery, University of Malaya Medical Centre, 50603 Kuala Lumpur, Malaysia
}

\begin{abstract}
We conducted a retrospective review on eleven patients who were treated for Type A and $\mathrm{C}$ distal femoral fractures (based on AO classification) between January 2004 and December 2004. All fractures were fixed with titanium distal femoral locking compression plate. The patient's ages ranged from 15 to 85 with a mean of 44 . Clinical assessment was conducted at least 6 months post-operatively using the Schatzker scoring system. Results showed that four patients had excellent results, four good, two fair and one failure.
\end{abstract}

Key Words:
Distal femoral fracture, Internal fixation, Distal femoral
locking compression plate

\section{INTRODUCTION}

Distal femoral fractures, especially AO Type C fractures are difficult to treat. Osteoarthritis may result when there is a diastasis of 3 or more millimetres. Previously the trend in treatment of these fractures leaned towards closed conservative management with traction, casting, or a combination of both. The problems associated with conservative management are the limitation of reduction and difficulty of maintaining reduction. Associated complications of prolonged immobilisation and economic considerations of increased hospital stay also limit their utility. External fixation with devices such as the hybrid external fixator and the Ilizarov external fixator are excellent for the treatment of comminuted fractures associated with bone loss. In addition to maintaining reduction whilst awaiting union, these devices can also be used to lengthen the bone. However, pin tract infections and joint contractures are common complications of these techniques.

As orthopaedic surgery has evolved, trends in treatment of supra-condylar and inter-condylar femoral fractures now more commonly involve operative management ${ }^{1}$. Internal fixation devices that have been used to treat these fractures include the $95^{\circ}$ angled blade plate, dynamic condylar screw plate, condylar buttress plate and retrograde supra-condylar inter-locking nail. However, as the complexity of fractures needing treatment has changed from simple extra-articular supra-condylar types to inter-condylar and metaphyseal comminuted types, these implants may not be ideal. Double plating, and more recently, locked plating techniques have been advocated ${ }^{2-5}$. However, with double plating, there is often extensive soft tissue stripping on both sides of the femur, resulting in reduced blood supply and potential nonunion and failure of the implants.

The Less Invasive Stabilization System (LISS) allows for fixed angle fixation of the distal femur and minimally invasive fixation of the femoral shaft ${ }^{6}$. However, there has been concern that the implant may be too stiff, and when associated with premature weight bearing by the patient, results in ultimate failure of the implant ${ }^{7}$.

Distal femoral locking compression plate (DF-LCP) has a smaller application device and allows both locking and compression screw fixation of the femur shaft. This study was conducted to examine the short term results, particularly early complications and healing rate of distal femoral fractures treated with the titanium distal femoral locking compression plate (Mathys Medical, Switzerland).

\section{MATERIALS AND METHODS}

This retrospective study was conducted in patients treated for distal femoral fractures in a single institution from 1 January 2004 to 31 December 2004.

\section{Technique}

Treatment of fractures was performed utilising the lateral approach in all patients. Fractures were reduced under direct vision using manual traction. A knee roll assisted the procurement and maintenance of reduction. The plate length, axial and rotational alignment were checked under image intensification. Temporary fixation was achieved through the use of Kirschner wires. Inter-condylar type fractures were converted to a single condylar block before DF-LCP 
fixation. Minimally invasive techniques were utilised where possible for insertion of proximal screws ${ }^{2}$. Where applicable, compression screws were used to approximate the plate to the femoral shaft.

\section{RESULTS}

The sample consisted of 11 patients with 12 DF-LCP devices implanted. Seven patients were male and four were female. (Table I) The patients' ages ranged from 15 to 85 years with a mean age of 44 years. The causes of fractures were motor vehicle accident in seven patients and a fall in four patients. There were no sports or industrial accidents. One patient sustained multiple fractures including lung injury which required ventilation. Seven fractures involved the right side and four involved the left. The average length of hospitalisation was 17.2 days with a range of 8 to 34 days. The average number of days from injury to surgery was 9.9 days with a range of 4 to 19 days. Patient follow-up ranged from 6 months to 15 months with an average of 9.7 months.

The operative time ranged from 80 minutes to 180 minutes, with an average of 119.2 minutes. The lengths of the plates used were 5 -holes ( 4 cases), 7-holes ( 5 cases), 9-holes (2 cases) and 11-holes (1 case).

According to the AO classification system, there were four Type A1, two Type A3, one Type C1, one Type C2 and three Type C3 fractures. Four (36.4\%) of the fractures were open with one sub-classified into Gustilo's IIIA and three IIIB. The seven $(63.6 \%)$ closed fractures were sub-divided according to Tscherne's classification (four type I and three type II).

Successful fracture union was defined as complete bridging callus in three cortices, together with painless full weight bearing. All patients were able to bear full weight postoperatively except for one patient. Excluding this patient, average time to union was 18 weeks with a range from 6 weeks to 36 weeks. Mean extension was $1^{\circ}$ (range $0^{\circ}$ to $5^{\circ}$ ), with mean flexion $107.7^{\circ}$ (range $40^{\circ}$ to $140^{\circ}$ ). Mean range of motion was from $1^{\circ}$ to $107.7^{\circ}$.
Using the Schatzker scoring system, there were four excellent results, four good, two fair and one failure'.

\section{Complications}

Complications included one implant fracture which required revision to another DF-LCP. There was one mal-alignment of the lower limb. One plate was cutting out due to poor fixation. There were no non-unions, deep infections or removal of implants due to ilio-tibial tract pain.

\section{Case example}

A 21 year old Chinese male was involved in a motor vehicle accident and sustained an open 3B, AO type 33A3 fracture of the left distal femur with a closed intra-articular distal end right radius fracture, closed mid-shaft right femur fracture, closed right 1st, 4th, 6th, 8th rib fractures with lung contusion.(Fig. 1a and $1 \mathrm{~b}$ )

Initial surgical management included wound debridement, tibial pin insertion for the left femur, open reduction and plating for the right radius and right femur. Definitive delayed open reduction and internal fixation with a 7-hole DF-LCP was performed for his left distal femur. (Fig. 2a and $2 b)$ He was discharged well with wheelchair ambulation and physiotherapy on an outpatient basis. At four months, he was fully bearing weight and radiographs revealed complete fracture healing. (Fig. 3a, 3b, 4a \& 4b)

\section{DISCUSSION}

Current fracture patterns veer towards complex comminuted types due to the prevalence of high speed vehicles. Improved healthcare results in a longer lifespan and subsequently presents us with more osteoporotic fractures which were previously treated using conservative methods.

The LCP is a single beam construct where the strength of its fixation is equal to the sum of all screw-bone interfaces rather than a single screw's axial stiffness and pullout resistance in unlocked plates ${ }^{8}$. Its unique biomechanical function is based on splinting rather than compression resulting in flexible stabilisation, avoidance of stress

Table I: Results for studied patients treated from January 2004 to December 2004

\begin{tabular}{|c|c|c|c|c|c|c|c|c|c|c|c|c|}
\hline No & age & sex & mech & $\begin{array}{l}\text { Soft } \\
\text { tissue } \\
\text { injury }\end{array}$ & Type & Complications & $\begin{array}{l}\text { bone } \\
\text { graft }\end{array}$ & $\begin{array}{l}\text { full wt } \\
\text { bear } \\
\text { (mths) }\end{array}$ & $\begin{array}{l}\text { LLD } \\
(\mathrm{cm})\end{array}$ & $\underset{\circ *}{\text { rom }}$ & $\begin{array}{l}\text { F/up } \\
\text { (mths) }\end{array}$ & $\begin{array}{c}\text { schatzke } \\
\text { score }\end{array}$ \\
\hline 1 & 45 & $\mathrm{~F}$ & MVA & O3B & $33 C 2$ & Implant fracture & Yes & 8 & 4 & $0-135$ & 15 & Good \\
\hline 2 & 35 & $M$ & MVA & O3B & $33 C 3$ & - & - & 9 & 2 & $5-40$ & 13 & Fair \\
\hline 3 & 80 & $\mathrm{~F}$ & Fall & $\mathrm{C} 1$ & $33 \mathrm{~A} 1$ & - & - & 6 & - & $0-120$ & 10 & Excellent \\
\hline 4 & 73 & $M$ & Fall & $\mathrm{C} 1$ & $33 \mathrm{~A} 1$ & - & Yes & 4 & - & $0-120$ & 8 & Good \\
\hline 5 & 85 & $\mathrm{~F}$ & Fall & $\mathrm{C} 1$ & $33 \mathrm{A3}$ & Plate cutting out & - & - & - & $0-45$ & 12 & Failure \\
\hline 6 & 25 & $M$ & MVA & O3A & $33 C 3$ & - & - & 2 & - & $0-135$ & 11 & Excellent \\
\hline 7 & 68 & $\mathrm{~F}$ & Fall & $\mathrm{C} 1$ & $33 C 3$ & Poor reduction & - & 5 & - & $0-90$ & 9 & Fair \\
\hline 8 & 19 & $M$ & MVA & $\mathrm{C} 2$ & $33 C 1$ & $\begin{array}{l}\text { Tibial pin site } \\
\text { infection }\end{array}$ & - & 3 & - & $0-110$ & 9 & Good \\
\hline 9 & 21 & $M$ & MVA & О3В & $33 \mathrm{A3}$ & Ventilated & - & 4 & - & $0-140$ & 8 & Excellent \\
\hline 10 & 18 & $M$ & MVA & $\mathrm{C} 2$ & $33 \mathrm{~A} 1$ & - & - & 3 & - & $0-140$ & 6 & Excellent \\
\hline 11 & 15 & $M$ & MVA & $\mathrm{C} 2$ & $33 \mathrm{~A} 1$ & - & - & 1.5 & - & $5-110$ & 6 & Good \\
\hline
\end{tabular}




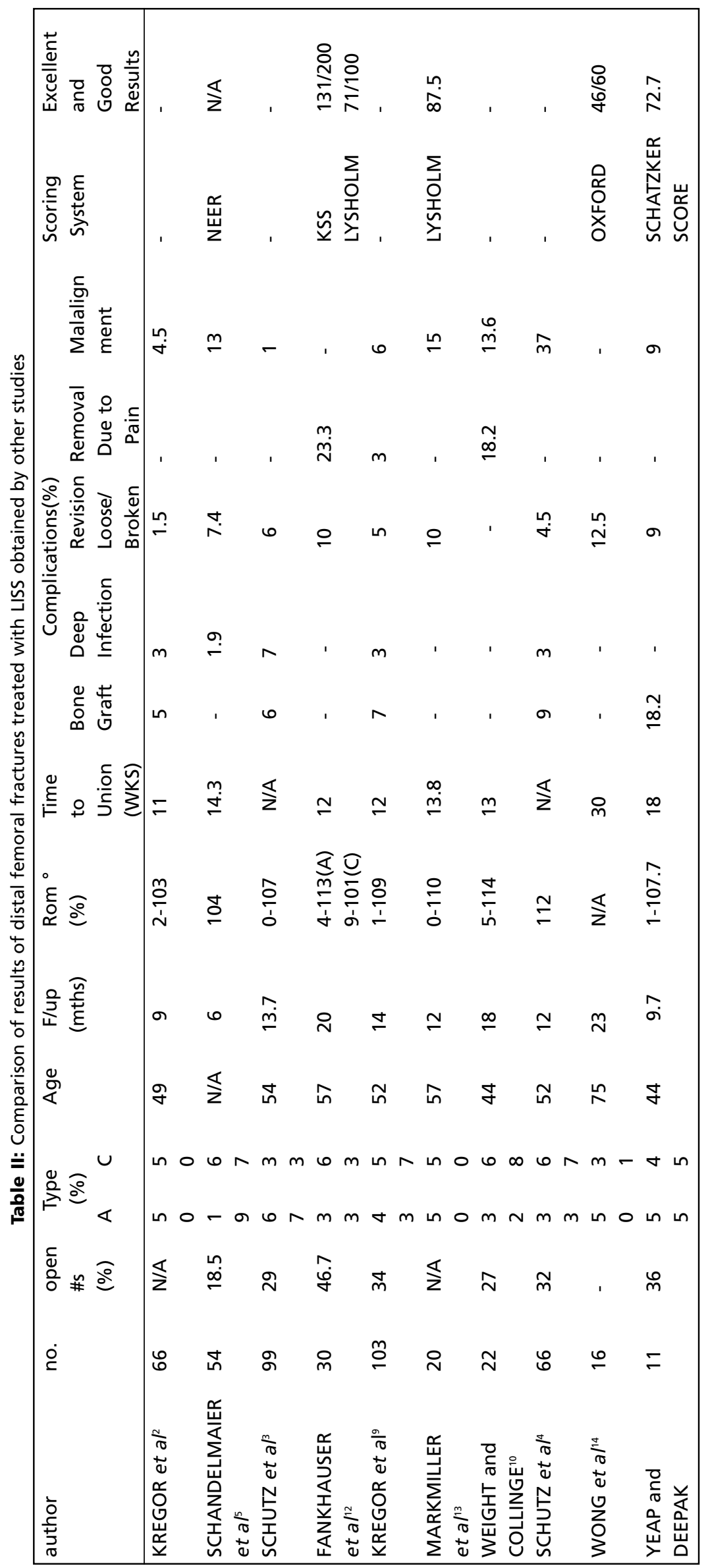


Distal Femoral Locking Compression Plate Fixation in Distal Femoral Fractures: Early Results

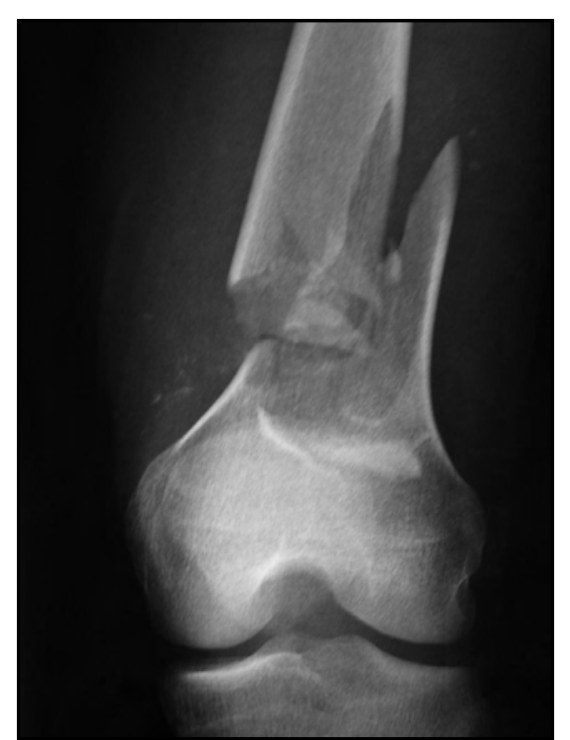

Fig. 1a: Radiograph of the left knee, preoperatively, AP view.

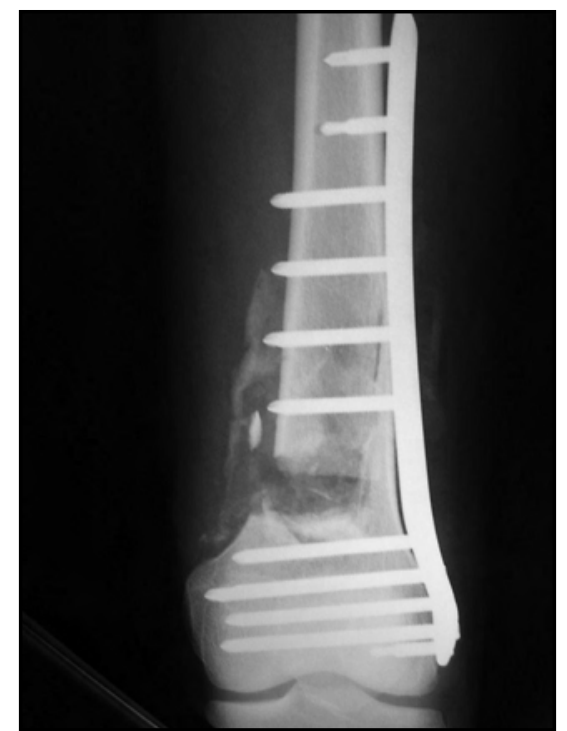

Fig. 2a: Radiographs of the left knee, AP view, initial postoperative period.

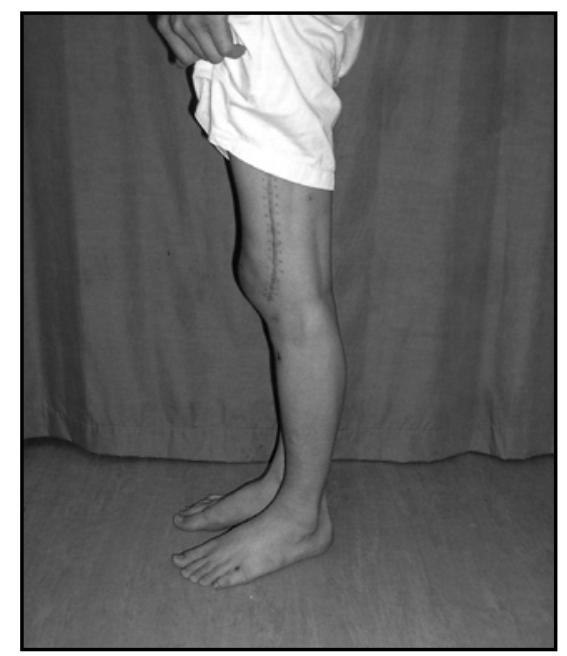

Fig. 3a: Full weight bearing, 4 months postoperatively.

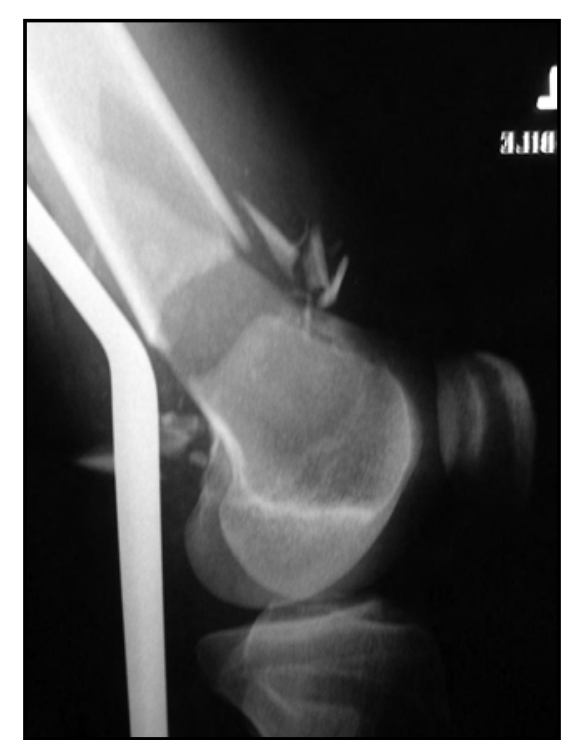

Fig. 1b: Radiograph of the left knee, preoperatively, lateral view.

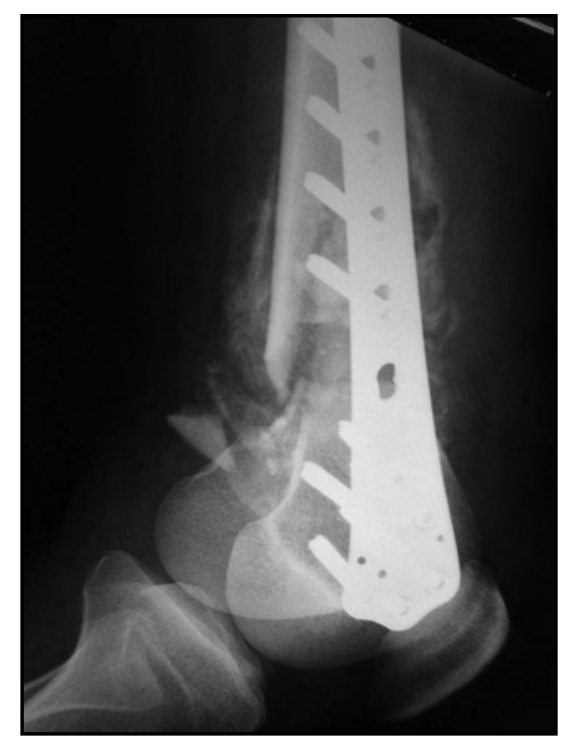

Fig. 2b: Radiographs of the left knee, lateral view, initial postoperative period.

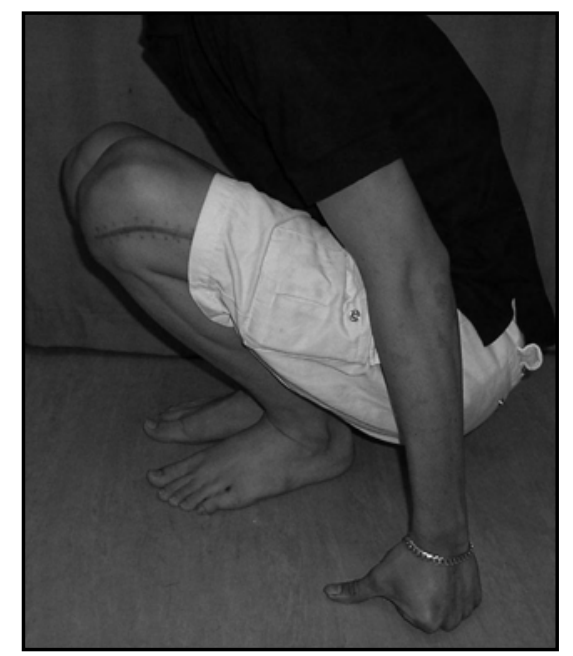

Fig. 3b: Full range of motion, 4 months postoperatively. 


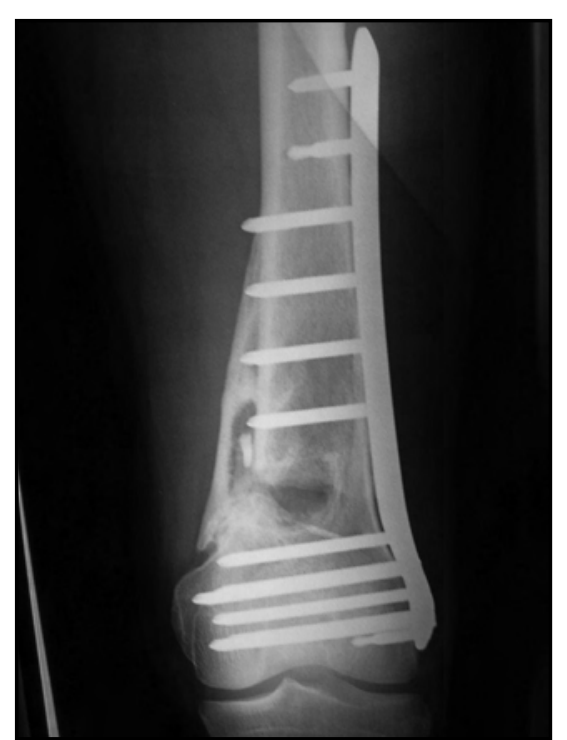

Fig. 4a: Radiograph of the left knee, AP view, 4 months postoperatively.

shielding and induction of callus formation'. When applied via a minimally invasive technique, it allows for prompt healing, lower rates of infection and reduced bone resorption as blood supply is preserved.

The DF-LCP is a further development from the LISS, which was introduced in the mid to late 1990 ' $\mathrm{s}^{5,6}$. The main difference between the DF-LCP and the LISS is that the LISS utilises an outrigger device for shaft holes, functioning essentially as a locking guide jig, which is attached to the distal part of the plate and guides the placement of the proximal locking screws. The shaft holes on the DF-LCP are oval allowing for the options of a compression screw or a locking screw. This leads to a more precise placement of the plate, as it is able to be compressed more closely to the bone.

Although DF-LCP is designed to fit the anatomy of the distal femur, we were worried about the fit in our local Asian population where shorter and smaller femurs are the norm. However, thus far, our limited numbers demonstrate that this is not an issue. Our cases do not demonstrate any irritation of the ilio-tibial tract which causes pain so severe that it necessitates removal of the implant ${ }^{9,10}$. This could be because we do not use the outrigger device, and are therefore better able to approximate the distal portion of the plate to the bone, ensuring that prominent hardware does not become an irritation to the ilio-tibial tract. Comparable studies utilising the LISS demonstrate similar short term results (Table II). Although the follow-up period of our series was short, studies have shown that early function is comparable to final long term outcome ${ }^{11}$. The outcome seems to correlate with fracture severity, anatomic reduction, aetiology, bone quality, length of time elapsed from injury to surgery, concomitant injuries , and exact positioning and fixation of the implant ${ }^{12}$.

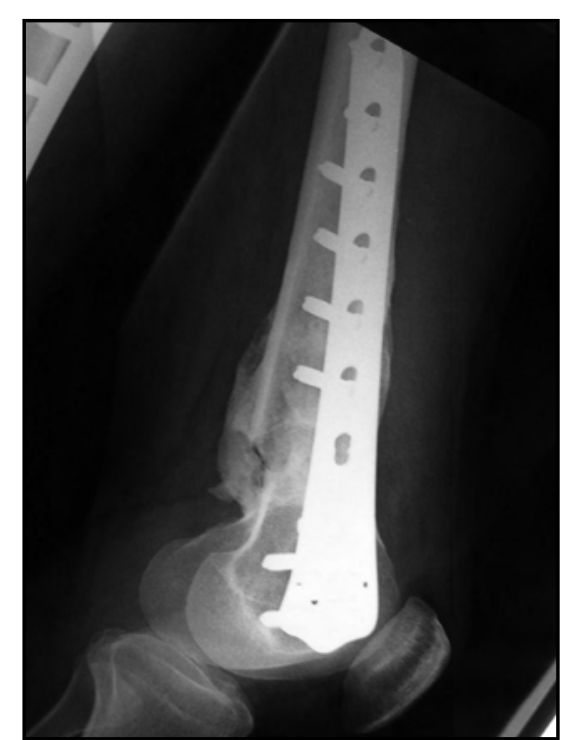

Fig. 4b: Radiograph of the left knee, lateral view, 4 months postoperatively.

The definitive long term prognosis remains unknown as of today, as the earliest LISS was implanted in the mid to late 1990's. Furthermore, the initial severe concomitant cartilage damage may predispose to early osteoarthritis although there is no evidence of that yet ${ }^{13}$.

In our series, two cases were scored as fair and one failed. These three cases required the use of aids and were either A3 or C3 type fractures. Previous studies have noted that type $\mathrm{A} 1$ and $\mathrm{C} 1$ fractures tend to have better outcomes ${ }^{11}$. The failure was in a severely osteoporotic female patient whose distal femoral fracture was poorly reduced leading to a situation in which the DF-LCP was cutting out. Furthermore, a short 5-hole plate was used. In osteoporotic bones, other authors recommend using a longer plate and bicortical anchoring on the shaft $f^{4,12,14}$. Loosening of the plate is usually due to misjudgement of the lever-arm or bone quality $^{12}$, but can also be due to improper positioning of the plate or screws, which was the case in this patient. Kregor et al recommends a small incision over the proximal part of the plate for accurate positioning, a technique that we utilised in later cases.

The two cases that scored 'fair' were type $\mathrm{C} 3$ fractures. One patient suffered from LCL and PCL instability postoperatively, but reported minimal pain when ambulating with a knee brace. There was varus angulation of $10^{\circ}$, internal rotation of $5^{\circ}$, and limb length discrepancy of 2 centimetres. In comminuted fractures such as this, the risk for rotation, varus, valgus and shortening is unavoidable ${ }^{12}$. The other case had a previous ipsilateral total hip replacement for a femoral neck fracture and the fracture was also not well reduced. 


\section{CONCLUSION}

The DF-LCP is a good implant to use for fractures of the distal femur. However, accurate positioning and fixation are required to produce satisfactory results. We recommend use of this implant in Type A and C, osteoporotic and periprosthetic fractures. Our early results are encouraging but long term studies are needed to prove definitively acceptable outcomes so that the technique can become part of the in the armamentarium of the orthopaedic trauma surgeon.

\section{REFERENCES}

1. Schatzker J, Lambert DC. Supracondylar Fractures of the Femur. Clin Orthop 1979; 138: 77-83.

2. Kregor PJ, Stannard J, Zlowodzki M, Cole PA, Alonso J. Distal femoral fracture fixation utilizing the Less Invasive Stabilization System (L.I.S.S.): The technique and early results. Injury 2001; 32: SC 32-47.

3. Schutz M, Muller M, Krettek C, et al. Minimally invasive fracture stabilization of distal femoral fractures with the LISS: A prospective multicenter study. Results of a clinical study with special emphasis on difficult cases. Injury 2001; 3 2: SC 48-54.

4. Schutz M, Muller M, Regazzoni P, et al. Use of the Less Invasive Stabilization System (LISS) in patients with distal femoral (AO33) fractures: a prospective multicenter study. Arch Orthop Trauma Surg 2005; 125(2): 102-8.

5. Schandelmaier P, Partenheimer A, Koenemann B, Grun OA, Krettek C. Distal Femoral Fractures and LISS Stabilization. Injury 2001; 32: SC 55-63.

6. Frigg R, Appenzeller A, Christensen R, Frenk A, Gilbert S, Schavan R. The development of the distal femur Less Invasive Stabilization System (LISS). Injury 2001; 32: SC 24-31.

7. Button G, Wolinsky P, Hak D. Failure of Less Invasive Stabilization System Plates in the Distal Femur:A Report of Four Cases. J Orthop Trauma 2004; 18(8): 565-70.

8. Egol KA, Kubiak EN, Fulkerson E, Kummer FJ, Koval JK. Biomechanics of Locked Plates and Screws. J Orthop Trauma 2004; 18: 488-93.

9. Kregor PJ, Stannard JA, Zlowodzki M, Cole PA. Treatment of Distal Femur Fractures Using the Less Invasive Stabilization System:Surgical Experience and Early Clinical Results in 103 Fractures. J Orthop Trauma 2004; 18(8): 509-20.

10. Weight M, Collinge C. Early Results of the Less Invasive Stabilization System for Mechanically Unstable Fractures of the Distal Femur (AO/OTA Types A2, A3, C2, and C3). J Orthop Trauma 2004; 18(8): 503-8.

11. Bolhofner BR, Carmen B, Clifford P. The Results of Open Reduction and Internal Fixation of Distal Femur Fractures Using a Biologic (Indirect) Reduction Technique. J Orthop Trauma 1996; 10(6): 372-7.

12. Fankhauser F, Gruber G, Schippinger G, et al. Minimal-Invasive Treatment of Distal Femoral Fractures with the LISS (Less Invasive Stabilization System): A Prospective Study of 30 Fractures with a Follow Up of 20 Months. Acta Orthop Scand 2004; 75(1): 56-60.

13. Markmiller M, Konrad G, Sudkamp N. Femur-LISS and Distal Femoral Nail for Fixation of Distal Femoral Fractures. Clin Orthop 2004; 426: 252-7.

14. Wong MK, Leung F, Chow SP. Treatment of Distal Femoral Fractures in the Elderly Using a Less-Invasive Plating Technique. Int Orthop 2005; 29: 117-20. 